\title{
The critical nature of the multidisciplinary management of breast cancer: Not just an academic institutional exercise
}

\author{
Mark Trombetta \\ Department of Radiation Oncology, Allegheny General Hospital, Allegheny Health Network, Pittsburgh, Pa. U.S.A. \\ Correspondence: Mark Trombetta. Address: Department of Radiation Oncology, Allegheny General Hospital, Allegheny \\ Health Network, Pittsburgh, Pa. U.S.A. 15212. Email: mtrombet@wpahs.org
}

Received: April 26, 2014

Accepted: April 26, 2014

Online Published: April 27, 2014

DOI : $10.5430 /$ jst.v4n3p1

URL: http://dx.doi.org/10.5430/jst.v4n3p1

\begin{abstract}
The complexity of the management of the cancer patient has reached such a level with a doubling rate of the knowledge base seemingly overnight, that even the most seasoned expert, well versed in the current literature, can be confounded. A prospective multidisciplinary approach can avoid missteps and instill confidence in the patient. Once a province of academic institutions alone, the multidisciplinary tumor board has taken a command role in the management of the cancer patient, whose care becomes increasingly complicated in the community setting. Common points of management are discussed here.
\end{abstract}

\section{Key words}

Radiation, Breast conservation, Mastectomy, Neoadjuvant, Multidisciplinary

\section{I ntroduction}

In many Academic Institutions, the multidisciplinary tumor board has been a staple of discussion and thought provoking inquiry many times provoking intense emotions, but always stimulating critical thought. When an academic program is robust, a multidisciplinary board should meet prospectively to discuss patient management in a conjoint and organized manner incorporating all opinions prior to major therapeutic decision making. In the community setting, prospective tumor board discussions are equally important, but logistical concerns many times make timely and prospective discussion difficult. Since approximately $85 \%$ of all cancer care is delivered in the community setting, the collaborative approach is critical. With the vast changes that occur sometimes in very short periods of time, in no disease site is prospective multidisciplinary management more important than in cancers of the breast.

\section{The proper indication for each imaging and diagnostic modality}

Since the role of mammography and ultrasound seemingly have been agreed upon, the use of the image-guided biopsy should be the routine. Initial diagnosis by excisional and incisional biopsies should be eliminated, and the use of fine 
needle aspiration and/or biopsy should be discouraged. Fine needle biopsies many times do not allow for adequate tissue sampling for diagnosis and incisional/excisional biopsies add nothing over core sampling in the initial diagnostic state. In addition, the performance of initial diagnostic incisional/excisional biopsies can undermine further surgical options, limiting future staged surgical procedures and negatively impacting cosmesis ${ }^{[1]}$. The collaborative interaction between surgeon, pathologist and diagnostic radiologist in this setting can improve outcomes.

Additionally, the routine use of magnetic resonance imaging (MRI) should be avoided and a judicious approach to selecting this modality should be employed. MRI should be reserved for those specific cases where the modality is necessary for further evaluation of the breast such as in women with dense breasts or conflicting physical findings not apparent on mammography or ultrasound imaging. In addition, evaluation of the contralateral breast in high risk patients [i.e. BRCA positivity] is a reasonable indication for MRI. Routine use of MRI has been associated with increased morbidity and higher unnecessary mastectomy rates ${ }^{[2]}$. A standardized multidisciplinary approach improves coordination of care and allows for the free flow of ideas leading to improved diagnostic outcomes potentially decreasing unnecessary and potentially confounding imaging. We reviewed a recent case where the interpretation of the mammogram read "dense breasts; Bi-Rads Category III". The patient had undergone a lumpectomy and the interpretation of the report by the consultant medical oncologist led to an MRI in a patient who was later classified as having "somewhat dense breasts" in a follow up a tumor board. An MRI was ordered which demonstrated questionable findings of low to intermediate suspicion. A follow up stereotactic biopsy was inconclusive and a lumpectomy was performed which demonstrated benign fibrous tissue. A lack of communication and the absence of a multidisciplinary review led to additional procedures, possible complications and a worsened cosmesis. As well, due to the lack of coordinated care the patient lost confidence in both the surgeon and the medical oncologist as she perceived missteps from both parties.

\section{Proper selection and sequencing of therapy; knowing the options}

The criticality of multidisciplinary management is most manifest in the delivery of therapy. As more and more patients are being treated with neoadjuvant therapy, coordination of care and integrated delivery remains paramount. One example relates to the delivery of neoadjuvant chemotherapy and hormonal therapy. While the clinical response of tumor shrinkage is gratifying, and the finding of a pathologic complete response gives excellent prognostic information, the primary endpoint of the neoadjuvant approach remains downsizing of the tumor to allow for breast conservation therapy. To date no randomized studies have demonstrated local control or survival benefit otherwise ${ }^{[3]}$. As well, the timing of hormonal manipulation has been questioned as related to radiotherapy. While no data have implicated these agents in the clinical sense, there are preclinical data which suggest that tamoxifen, for example, may halt cancer cells in a relatively radio-resistant cell cycle phase ${ }^{[4]}$. Coordination of care between the radiation and medical oncologist is critical in order to deliver a unified message to the patient regarding these and other issues.

Additionally, the surgical management should be considered in the context of a multidisciplinary forum. Recently the overall medical community has regressed in the area of breast preservation. Mastectomy has become more frequently discussed despite data that do not show the necessity of more aggressive surgical management. The media have helped negatively change this perception and advocates for prophylactic mastectomy have influenced countless women. The shock of initial diagnosis many times leads to hasty decision making on the part of the patient who may later regret such a choice since body self-image suffers greatly in many women after mastectomy ${ }^{[5,6]}$.

Even those patients thought to be candidates for mastectomy from a traditional standpoint can safely undergo breast conserving therapy with careful whole breast or accelerated partial breast radiation. For example, patients with ipsilateral pacemakers have recently been shown to safely undergo breast preservation ${ }^{[7]}$. Patients who develop ipsilateral breast cancer recurrences; once an absolute indication for mastectomy, have been safely and successfully retreated and their 
breasts preserved ${ }^{[8-10]}$. Patients with connective tissue disorders such as lupus erythematosis are many times considered to be poor radiation candidates due to excessive skin reactions, but reported safe treatment has been reported in selected women who have not had major exacerbations of skin reaction previously ${ }^{[11]}$. Thoughtful planning and careful patient counseling with a thorough discussion of the alternatives can greatly assist women. Multiple consecutive discussions may be helpful for patients initially overwhelmed by the diagnosis, and not able to completely think objectively. In this setting, multidisciplinary consultation may serve as a natural time buffer to aid patient decision making in an objective manner.

In summary, the multidisciplinary management of breast cancer is critical to the proper diagnosis and management of these patients and will help to instill patient confidence in the team of physicians privileged to care for them.

\section{References}

[1] White RR, Halperin T, Olson J, et al. Impact of core biopsy on the surgical management of mammographic abnormalities. Ann Surg. 2001 Jun; 233(6): 769-77. PMid:11371735 http://dx.doi.org/10.1097/00000658-200106000-00006

[2] Pilewskie M, King T. Magnetic resonance imaging in patients with newly diagnosed breast cancer: A review of the literature. Cancer. 2014 Apr 18 [Epub ahead of print]. Available from: http://onlinelibrary.wiley.com/doi/10.1002/cncr.28700/abstract

[3] Mamounas E, Anderson S, Dignam JJ, et al. Predictors of loco-regional recurrence after neoadjuvant chemotherapy: results from combined analysis of National Surgical Adjuvant Breast and Bowel Project B-18 and B-27. J Clin Oncol. 2012 Nov 10; 30(32): 3960-6. PMid:23032615 http://dx.doi.org/10.1200/JCO.2011.40.8369

[4] Chargari C, Toillon R, Macdermed D et al. Concurrent hormone and radiation therapy in patients with breast cancer: What is the rationale? Lancet Oncol. 2009 Jan; 10(1): 53-60. http://dx.doi.org/10.1016/S1470-2045(08)70333-4

[5] Ganz PA, Schag CA, Lee JJ, et al. Breast conservation versus mastectomy: Is there a difference in psychosocial adjustment or quality of life in the year after surgery? Cancer. 1989; 69: 1729-1738.

http://dx.doi.org/10.1002/1097-0142(19920401)69:7<1729::AID-CNCR2820690714>3.0.CO;2-D

[6] Ganz PA, Cascarelli A, Fred C, et al. Breast cancer survivors: Psychosocial concerns and quality of life. Breast Cancer Res Treat. 1996; 38: 183-199. PMid:8861837 http://dx.doi.org/10.1007/BF01806673

[7] Croshaw R, Kim Y, Lappinen E, et al. Avoiding mastectomy: Accelerated partial breast irradiation for breast cancer patients with pacemakers or defibrillators. Ann Surg Oncol. 2011; 18(12): 3500-3505. PMid:21607774 http://dx.doi.org/10.1245/s10434-011-1784-2

[8] Trombetta M, Julian T B, Kim Y, et al. Mature follow-up of low dose rate brachytherapy following ipsilateral breast tumor recurrence in patients initially treated with breast conservation therapy. J Solid Tumors. 2012; 2(1): 8-15. http://dx.doi.org/10.5430/jst.v2n1p8

[9] Chadha M, Trombetta M, Boolbol S, et al. Managing a small recurrence in the previously irradiated breast: Is there a second chance for breast conservation? Oncology. 2009; 23(11): 933-40. PMid:19947343

[10] Hannoun-Levi JM, Ihrai T, Courdi A. Local treatment options for ipsilateral breast tumor recurrence: Cancer Treat Rev 2013 Nov; 39(7): 737-41. PMid:23465859 http://dx.doi.org/10.1016/j.ctrv.2013.02.003

[11] Patel A, Hallemaier C, Petersen I, et al. Acute and late toxicities of radiotherapy for patients with discoid lupus erythematosis: a retrospective case-controlled study. Radiat Oncol. 2012 Feb 16; 7-22. 